\title{
RESIDUOS DE COCO. ARTE Y VERMICOMPOSTADO PARA SU REUTILIZACIÓN
}

\author{
Maria Desamparados Soriano a , Francisco García b', Laura Garcia-España c \\ a Departamento de Producción Vegetal. Universitat Politécnica de Valencia. asoriano@prv.upv.es

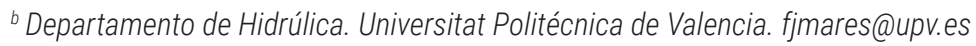 \\ c Departamento de Biología Vegetal. Universitat de Valencia. laugars2@alumni.uv.es
}

\begin{abstract}
Coconut waste is difficult to eliminate and although it is useful as a substrate in gardening, its long life makes it useful for other activities, being widely used within the art to make crafts and decorative works both in jewelry, ornaments, instruments, etc.. In addition, some parts of the coconut with difficult reuse after agro-industrial use can be processed by composting transforming it into a useful resource as a fertilizer, given its high proportion of nutrients. Its usefulness in art is mentioned and the results related to the reuse of coconut waste from a start-up in Ecuador after the agro-industrial use of coconut are presented. The composted waste was prepared by adding rabbit manure and water, and analyzed at different stages of the composting process. During the waste transformation process, parameters such as temperature and humidity were controlled, a high temperature is required to disinfect the final product and maintaining humidity around $60 \%$ to facilitate the process. The results show the nutrient richness of the final product, suitable for use as a fertilizer, having begun trials to be used also as a substrate $v$ for plant development. The study is part of a Cooperation Project of the Polytechnic University of Valencia in the School of Agronomy and Rural Environment.
\end{abstract}

Keywords: coconut plant, waste, vermicompost, resources.

Resumen: Los residuos de coco son difíciles de eliminar y aunque presentan utilidad como sustrato en jardinería, su larga vida los hace útil para otras actividades, siendo muy utilizado dentro del arte para realizar manualidades y obras decorativas tanto en joyeria, adornos, instrumentos, etc. Además algunas partes del coco con dificil reutilización tras u uso agroindustrial puede procesarse por compostado transformándolo en un recurso útil como fertilizante, dada su alta proporción en nutrientes. Se menciona su utilidad en el arte y se presentan los resultados relativos a la reutilización de residuos de coco procedentes de una empresa emergente de Ecuador después de la utilización agroindustrial del coco. Los residuos compostados se prepararon adicionando estiercol de conejo y agua, y se analizaron en diferentes etapas del proceso de compostaje. Durante el proceso de transformación del residuo se controlaron parámetros como la temperatura y la humedad, se precisa una temperatura elevada para realizar la desinfección del producto final y manteniendo la humedad alrededor del $60 \%$ para facilitar el proceso. Los resultados muestran la riqueza en nutrientes del producto final, apto para su utilización como fertilizante, habiéndose iniciado los ensayos para poder utilizarse también como sustrato v para el desarrollo vegetal. El estudio se enmarca dentro de un Proyecto de Cooperación de la Universitat Politécnica de Valencia en la Escuela de Agronomia y del Medio Rural.

Palabras clave: planta de coco, residuos, vermicompostaje, recursos.

Citar como: Soriano, M. D., García, F., Garcia-España, L. (2022). Residuos de coco. Arte y vermicompostado para su reutilización. En Actas del III Congreso Internacional sobre Patrimonio Alimentario y Museos. 25-26 noviembre, 2021, Valencia, España. pp. 281-290. https://doi.org/10.4995/EGEM2021.2021.14899 


\section{Introducción}

La reutilización de los residuos es una investigación dirigida hacia la gestión, tratamiento y aprovechamiento de los residuos agroindustriales para la producción de productos útiles como insumos de otros procesos industriales, ha sido y está siendo uno de los temas prioritarios en los campos de la biotecnología, agroquímica y ciencias medioambientales. Por otro lado, las normativas europeas, nacionales y autonómicas, incorporan capítulos específicos sobre los residuos derivados de la actividad agrícola y ganadera dado que su tratamiento requiere especificidades propias, debido a sus posibles orientaciones estratégicas. La detección de contaminantes emergentes en todos los compartimentos ambientales, así como en muchos residuos, ha creado, en los últimos años, la necesidad de desarrollar procedimientos para su eliminación que puedan aplicarse como técnicas de biorremediación. La utilización de los procesos de compostaje se emplean como estrategias para el aprovechamiento y el reciclado de residuos orgánicos y, por ende, para el tratamiento y valorización de los biorresiduos agrícolas; esto puede proporcionar enmiendas orgánicas que mejoren la calidad del suelo y favorezcan el desarrollo vegetal. La necesidad de mejorar, proteger y conservar la calidad de los suelos y su funcionalidad con el fin de incrementar la producción y la seguridad alimentaria, junto con el reciclado de residuos, constituyen una de las bases que marcan el camino hacia la sostenibilidad y contribuyen, en gran parte, a llevar a cabo de manera exitosa las directrices de la economía circular, tanto en el presente como en el futuro.

Actualmente de los residuos agroindustriales del coco sólo se aprovecha el $17 \%$ de la cubierta y parte media de los cocos, siendo el $83 \%$ restante considerado como residuo.

En arte el coco ha sido utilizado para manualidades desde hace años, llegando a encontrarse verdaderos museos del coco. En estos museos se pueden ver fotografias, informaciòn sobre la palma de coco y sus derivados, artesanías elaboradas con materiales extraídos de la planta, asi como herramientas y utensilios que se usan como decoración o en cocina, etc.

Por otra parte, cada vez en mayor medida se buscan fertilizantes orgánicos que actuén mejorando la fertilidad del suelo, sobre sus propiedades físicas, químicas y biológicas y mejoren

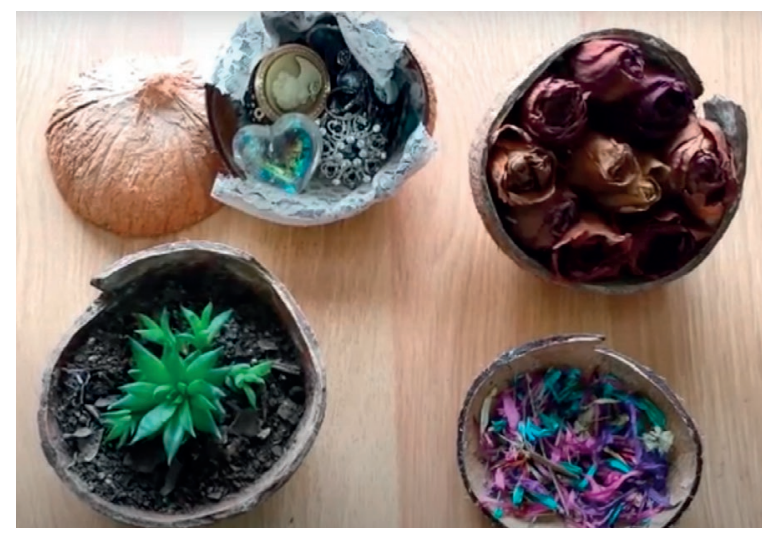

Figura 1. Reutilización de la cascara de coco.

Fuente: https://www.bing.com/videos/search?q=artesania+en+coco+museos\&\&view=detail\&mid= 3FBA7F93C283008070FC3FBA7F93C283008070FC\&\&FORM=VDRVR 


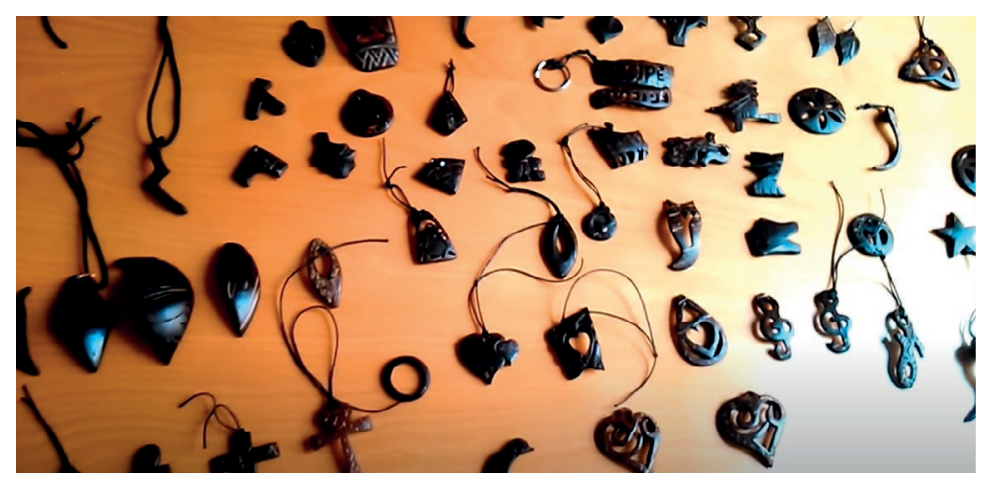

Figura 2. Reciclando cáscara de coco! - Bing video. Artesania con cascara de coco de Antonio Palazón. Fuente: https://www.youtube.com/watch?v=aeb3ilESVc4V.

I.a pérdida de materia orgánica del suelo Actualmente se plantea un escalamiento del proceso de aprovechamiento de los residuos del coco que beneficie a las comunidades que lo cultivan.

La obtención de materia orgánica a partir de residuos minimiza el daño medioambiental apuntando hacia una agricultura más sostenible. Siendo el proceso de compostaje una interesante alternativa que facilita el reciclado y reutiliza los residuos convirtiéndolos en compost.

Por todo lo anterior, el presente trabajo pretende dar una visión de su reutilización tanto en arte y como fertilizante de este residuo por su utilidad en ambos campos.

\section{Metodología}

\subsection{Material vegetal utilizado}

El coco es el fruto de Cocus nucifera (familia Arecaceae), se trata de un árbol originario de Asia. Se comercializa como fruta fresca o destinado con fines industriales, y se cosecha entre los 5 y 6 meses. Posee elevado contenido de agua y azúcares.

Existe gran cantidad de variedades. Se desarrolla en terrenos arenosos y salinos, tolerante al viento necesitando un clima sueve para su crecimiento. Su fruto presenta propiedades medicinales, y nutricionales, presentando una gran utilidad desde el punto de vista artesanal.

\subsection{Preparación del experimento}

Se utilizan residuos de coco para su transformación en un experimento de compostaje. En una primera fase se seleccionaron los residuos de coco de la parte exterior de la corteza, media e interior, Se trituraron utilizando un molino de cuchillas (Biotriturador VIKING 4000), hasta alcanzar un tamaño aproximado de $1 \mathrm{~cm}$ de longitud (Figura x). Los residuos triturados se mezclaron con estiércol de conejo parcialmente transformado procedente de las granjas de la ETSIAMN. Las proporciones de las mezclas fueron 1:1:1 (residuo, estiércol, agua). Las mezclas se homogeneizaron introduciendo una cantidad de $25 \mathrm{~kg}$ en recipientes de PVC de 32 litros. Se habilitaron tres recipientes para el compostaje, para realizar repeticiones suficientes. Seguidamente se les añadió agua a las muestras preparadas para compostaje en cantidad suficiente para lograr una humedad del $70-85 \%$. En 

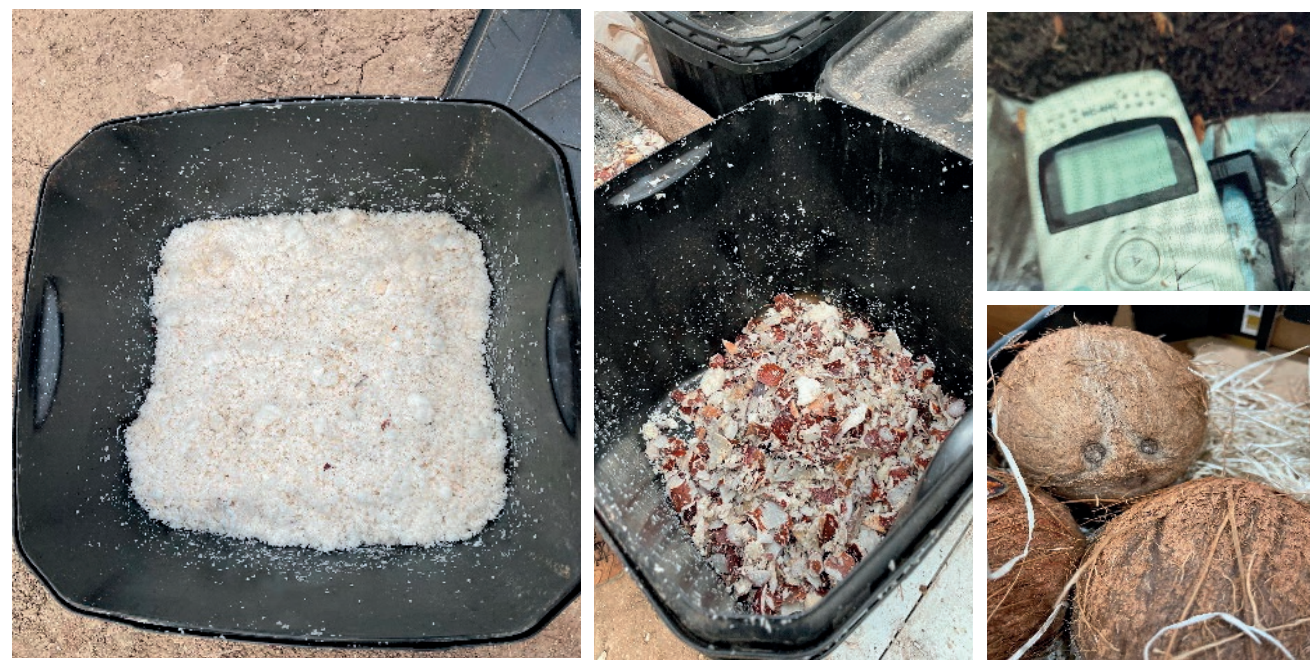

Figura 3. Residuos de coco. Termómetro

Fuente propia.

las mezclas preparadas para compostar, la humedad se mantuvo siempre por encima del 50\%, y nunca bajando del valor crítico a partir del cual la actividad biológica podría detenerse según indican autores como Moreno y Moral (2008). Finalmente se taparon los recipientes dejándolos en condiciones de oscuridad y con una temperatura inicial entre 25 y $29^{\circ} \mathrm{C}$. Para realizar todas estas experiencias se habilitó una planta en los recintos de la Escuela Técnica Superior de Agronomía de la Universidad Politécnica de Valencia dedicada al tratamiento de los residuos. Durante 4 meses se controló la humedad y la temperatura. Se voltearon las pilas con una frecuencia semanal para asegurar una correcta aireación de éstas y mantener la humedad.

Al cabo de este periodo se tomaron las muestras, se secaron y analizaron realizando determinaciones como el contenido en materia orgánica, nitrógeno total y análisis elemental (Métodos Oficiales Ministerio Agricultura, 1991). Se utilizó un microscopio electrónico de barrido HITACHI mod. S-4100 con sistema de captación de imágenes EMIP 3.0 y sistema de microanálisis RONTEC f) cañón de emisión de campo con detector BSE AUTRATA.

\section{Resultados y Discusión}

\subsection{Utilidad y uso del coco}

El coco presenta gran utilidad en industrias de alimentos, cosméticos, textil, etc. Al mismo tiempo que por su contenido en aceite, resistente a altas temperaturas y con elevado contenido en grasas saturadas. El agua de coco contiene minerales, vitaminas, proteínas, carbohidratos y antioxidantes, siendo una bebida isotónica e hidratante.

La cáscara se utiliza en los cultivos como abono, pudiendo ser además aprovechado para el ganado. Contiene ácido laúrico que se utiliza para producir jabones, detergentes y champús. También se utiliza como fragancia y cosméticos. 


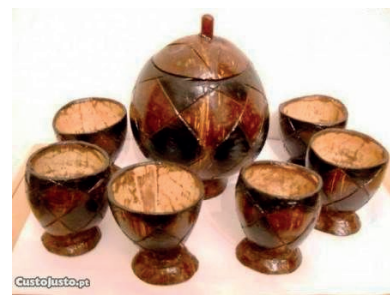

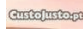
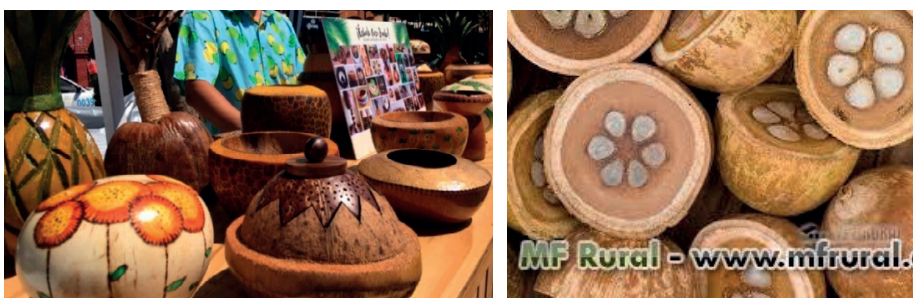

Figura 4. Museos del coco.

Fuente: https://www.mfrural.com.br/.

Como propiedades medicinales tiene a regular la presión sanguínea, los niveles de azúcar y colesterol, además tiene propiedades antibacterianas, antifúngicas, antiinflamatorias y antisépticas, además presenta un alto grado de protección solar.

El coco ha sido utilizado para manualidades desde hace años, en todas las partes del coco pulpa, corteza y malla, llegando a encontrarse verdaderos museos del coco. En estos museos se pueden ver fotografías, información sobre la palma de coco y sus derivados, artesanías elaboradas con materiales extraídos de la planta, así como herramientas y utensilios que se usan como decorativos o en cocina, etc. Igualmente se utilizan para elaborar artesaria decorativa de tipo pendientes y pulseras.

\subsection{Producto final del compostaje}

El contenido de C se encuentra alrededor del 20\%. Valores elevados si comparamos con otros residuos como el palmito (7\%) o el arroz (3\%). La pulpa del coco seco contiene más de un $60 \%$ de aceite. Los residuos de coco poseen proteínas con un promedio del $22 \%$ de proteína.

Con un contenido en materia seca del $92 \%$, un $22 \%$ de proteína cruda y un $16 \%$ de cenizas. Este valor destaca si lo comparamos con otros residuos como el arroz con un valor medio de proteína tan solo alrededor del 10\%.

La Tabla 1 muestra los resultados de la caracterización de los residuos de coco así como del estiércol de conejo (EC), y en la Tabla 2 se indica el manejo de los residuos, humedad, pH y relación $\mathrm{C} / \mathrm{N}$ de las mezclas.

Desde el punto de vista del compostaje hay que señalar que el residuo de coco posee un contenido de humedad y una relación $\mathrm{C} / \mathrm{N}$ elevada, para obtener una mezcla adecuada se requiere un residuo que pueda aportar elevados nutrientes, en este sentido, el estiércol de conejo aporta un contenido importante de nitrógeno (1,5\%) que no tiene nuestro residuo de coco $(0,6-1,0)$.

Aunque el compostaje de este residuo por si solo puede ser factible, se recomienda su mezcla con otros residuos que contengan características adecuadas de manera que se acelere y mejore el rendimiento del proceso de compostaje. Existen muchas referencias en la bibliografía en las cuales se lleva a cabo técnicas de tratamiento con residuos de poda (Day y Shaw, 2001; Goldstein, 2004; Smidt., 2009; Haug, 2014; Abbasi et al., 2015; Rekasi et al., 2019).

Los contenidos de humedad del residuo son muy elevados situándose entre 80 y $85 \%$ mientras que el contenido del estiércol de conejo es ligeramente inferior (48,8\%). Como es obvio, la salinidad de los estos residuos agrícolas es baja (entre 0,3 y 0,8 dS. $\mathrm{m}^{-1}$ ) y la del estiércol de 
conejo es bastante más elevada $\left(2,1 \mathrm{dS} \cdot \mathrm{m}^{-1}\right)$. El pH del residuo de coco es de 5,9. El carbono total varía alrededor del $20 \%$ estando la relación C/N inicial en 45,3.

Parámetros como son los nutrientes tales como fósforo, potasio, calcio y magnesio, presentan los valores característicos de residuos agrícolas y complementan el medio nutritivo adecuado para los organismos que deben llevar a cabo el proceso. Respecto al sodio, indicar de la misma manera que es muy bajo para los residuos agrícolas (de 0,1 a 0,03\% de $\mathrm{Na}_{2} \mathrm{O}$ ) y más elevado para el estiércol de conejo (1,8\%). Respecto al residuo de conejo nuestros valores son semejantes a los mostrados en los trabajos de Soliva, (2001); Gong et al., (2018); Li et al., (2013); Boluda et al., (2014); Lim et al., (2016).

Tabla 1. Caracterización analítica de los residuos agrícolas (entre paréntesis se indica la desviación estándar, n=3).

\begin{tabular}{lcc}
\hline Parámetro & R. Coco & Estiércol de conejo \\
\hline Humedad \% & $75,3(0,4)$ & $48,8(2,4)$ \\
CE $\left(\right.$ dS. m $\left.^{-1}\right)$ 1:10 & $0,7(0,1)$ & $2,1(0,0)$ \\
pH 1:10 & $5,7(0,0)$ & $8,2(0,0)$ \\
Carbono Total $(\%)$ & $42,2(1,1)$ & $38,6(0,8)$ \\
Relación C/N & $42,3(1,0)$ & $25,7(0,6)$ \\
\hline
\end{tabular}

Después de aproximadamente 4 meses tras haber iniciado el proceso se consideró finalizado y se procedió a la evaluación de las características del compost obtenido (Tabla 2).

Tabla 2. Características físicas y químicas del compost obtenido (entre paréntesis se indica la desviación estándar, n=3).

\begin{tabular}{lc}
\hline Parámetro & Coco \\
\hline Humedad \% & $45(0,2)$ \\
Densidad (g /cm3) & $0,8(0,1)$ \\
CRA \% & $85(2,9)$ \\
CE $\left(\mathrm{dS} . \mathrm{m}^{-1}\right)$ 1:10 & $1.9(0,1)$ \\
pH 1:10 & $7,8(0,1)$ \\
Carbono Total (\%) & $23,5(0,1)$ \\
Relación C/N & $14,5(0,4)$ \\
CIC $\left(\mathrm{cmol}_{.} \cdot \mathrm{kg}^{-1}\right)$ & $44,2(2,0)$ \\
\hline
\end{tabular}

El estudio del análisis elemental muestra la abundancia de silicio, potasio y calcio como constituyentes principales seguidos de otros macronutrientes como aluminio, sodio y magnesio (Figura 5).

\subsection{Microscopía electrónica y Espectroscopia infrarrojo de transformada de Fourier (FTIR)}

El estudio de las características de los residuos permite por IR la identificación de los grupos químicos que muestran los cambios que se producen durante el proceso de compostaje (Figura 5). 

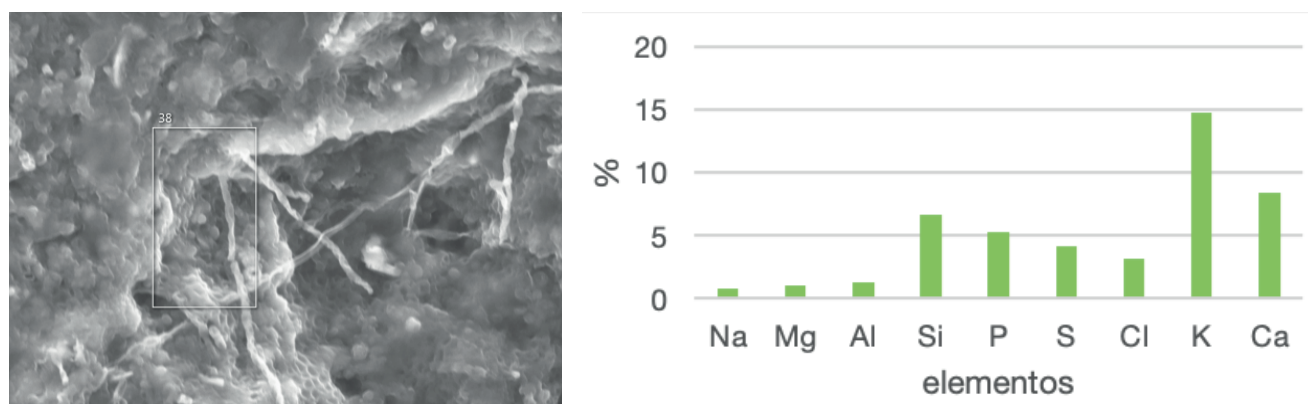

Figura 5. Imágenes SEM del residuo de coco. Resultado del análisis elemental del residuo de coco.

Al analizar los espectros obtenidos se asignaron los picos a los grupos funcionales presentes en los materiales de acuerdo a sus respectivas señales. La banda más amplia alrededor de los 3000 a 4000 cm-1 corresponde al estiramiento del grupo hidroxilo debido al enlace de hidrógeno de los compuestos poliméricos como alcoholes, fenoles y ácidos carboxílicos típicos de la celulosa y la lignina. Los picos entre 1720 y $1730 \mathrm{~cm}^{-1}$ son debidos a la vibración del estiramiento del enlace CO producido por grupos carboxílicos iónicos o sus esteres. Alrededor de 1600 a 1400 $\mathrm{cm}^{-1}$ se muestran las vibraciones delos grupos carboxílicos, siendo además la señal de 1600 $\mathrm{cm}^{-1}$ corresponde con las vibraciones del doble enlace $\mathrm{C}=\mathrm{C}$ del anillo aromático de la lignina. La señal en $1033 \mathrm{~cm}^{-1}$ es característico de los grupos de la celulosa. El estudio de la evolución del proceso permite observar la disminución de las bandas propias de grupos aromáticos por transformación del residuo así como la disminución de celulosa y grupos $\mathrm{OH}$. Las fotografías obtenidas por microscopia muestran la evolución y transformación de las fibras del residuo y la mineralización de la muestra.

\section{Conclusiones}

La planta de coco ha sido utilizada desde antiguo por sus diversas funciones estando relacionada desde antaño con el arte, siendo numerosas sus aplicaciones en manualidades y obras de arte, encontrándose muestras en museos y exposiciones a lo largo de la historia.

La reutilización de residuos por compostado ha supuesto un nuevo campo para transformarlo en recurso, al obtener un producto apto para su utilización como abono por su riqueza en materia orgánica y nutrientes.

Tanto los ejemplos recogidos en museos como la comercialización de los productos obtenidos indican la utilidad de esta planta en diversos medios, que pueden introducirse además en el ámbito docente en los estudios de Bellas Artes y en el estudio y aplicación agronómica de residuos.

\section{Agradecimientos}

Trabajo realizado en el marco del Proyecto de Cooperación de la Universitat Politècnica de València. Programa Adisedo Cooperación. Años 2020-2022. «TíTULO DEL PROYECTO: Evaluación de la sostenibilidad del establecimiento de una fábrica artesana de productos de coco en la subregión de Sanquianga (departamento de Nariño, Colombia)». 


\section{Bibliografía}

ABBASI, S. A., NAVEEM, M., ABBASI, T. ( 2015). "Vermicomposting of phytomass: limitations of the past approaches and the emerging directions". En: Journal of Cleaner Production. 93, pp. 103-114.

HUANG K, LI FS, WEI YF, FU XY, y CHEN, XM. (2014). "Effects of earthworms on physicochemical properties and microbial profiles during vermicomposting of fresh fruit and vegetable wastes". En: Bioresour Technol., 170, pp. 45-52. https://doi.org/10.1016/j.biortech.2014.07.058 PMID: 25118152.

REKASII, M., MAZU, N., DRASKOVITS, E., BERNHARDT, B., SZABO, A., RIVIER, P.A., FARKASS, C., BORSANYI, B., PIRKO, B. y MOLNAR, S. (2019). "Comparing the agrochemical properties of compost and vermicomposts produced from municipal sewage sludge digestate". En: Bioresource Technology, 291, 121861, https://doi.org/10.1016/j.biortech.2019.121861.

YONG, WJWH. (2009). "Chemical composition and biology in cooconut (Cocos nucifera L.)" Molecules: 14 , pp. 5144-5164. 